\title{
Design criteria for novel supersonic nozzles with high pitch-chord ratio
}

\author{
Maksim Smirnov 1,*, Aleksandr Kirillov ', Kirill Lapshin ', Gennadii Porshnev ', and \\ Aleksandr Laskin ${ }^{1}$
}

${ }^{1}$ Peter the Great St. Petersburg Polytechnic University, 195251 Saint-Petersburg, Russia

\begin{abstract}
High pitch-chord ratio turbine stages are a novel class of high enthalpy drop impulse turbines. Significant limitation of traditional design approach for such turbines is a low partial admission rate due to low volumetric flow rates. The main idea of high pitch-chord ratio design is in increase of the stage load coefficient with decrease of the flow angles. The latter allows to provide higher partial admission rate and, hence, higher stage efficiency compared with the traditional design approach. As a consequence, efficiency of the high pitch-chord ratio stages varies between $65 \%$ and $75 \%$, whereas partially admitted stages efficiency tend not to exceed $60 \%$. High pitch-chord ratio nozzles have a rectangular cross section. This feature leads to a vortex pair occurrence in a nozzle flow path. An interaction of these vortices for trans- and supersonic stages leads to additional losses and comparably low nozzle efficiency. The present paper aimed at first, establishing the design criteria which are essential for the nozzle efficiency, and, second, finding the optimal ratios of the proposed criteria. CFD simulation approach is used for the study. The gained deliverables are helpful for increasing of the nozzle velocity ratio.
\end{abstract}

\section{Introduction}

Development of modern propulsion systems for transport, marine and space applications leads to new requirements for weight-size parameters and cost effectiveness for such installations. Turbines are widely used as a drive for such units and, hence, are capable to be improved in terms of their weight-size parameters and efficiency. Decreasing of turbine weight-size parameters is related with decreasing of its stages number and consequent increase of a load per stage. The last obstacle inevitably tends to a growth of a pressure ratio per stage, transonic / supersonic flow regime occurrence and, hence, dramatic decreasing of a stage efficiency. Therefore, the special design of the nozzle vanes and rotor blades is required to fulfill the new spatial and cost effectiveness limitations.

Highly loaded turbines may have both impulse and reaction design and often have partial admission as in the works of Kurzrock (1989) [1], Kunte and Seume (2013) [2]. Investigations of supersonic axial impulse turbines with axisymmetric nozzles and partial admission provided by Natalevich (1979) [3], Dorney et al. (1999) [4], Tog and Tousi

\footnotetext{
* Corresponding author: m.smirnov.turbo@mail.ru
} 
(2013) [5], Varma and Soundranayagam (2012) [6] show that efficiency of these turbines does not exceed $65 \%$. Investigation of the Curtis stage with partial admission, which first row belongs to highly loaded class, provided by Rashid et al. (2007) [7], showed that efficiency of such stages does not exceed $60 \%$. Highly loaded transonic reaction cascades were investigated by Sonoda et al. (2006) [8] and Wolf et al. (2010) [9]. Rajeevalochanam et al. (2016) [10] numerically estimated efficiency of high-loaded axial reaction turbine as $88 \%$.

High-loaded impulse stages are commonly used thanks to their simplicity compared with reaction stages. Typically, these stages have a nozzle outlet angle $\alpha_{1}$ and rotor inlet angle $\beta_{1}$ higher than $15^{\circ}$ (see, e.g., Aungier (2006) [11], Kirillov (1972) [12]). However, decreasing of $\alpha_{1}$ angle provides increasing of a circumferential load per stage according to the Banki equation:

$$
H_{u}=2 \varphi H_{0} \frac{u}{C_{0}}\left(\cos \alpha_{1}-\frac{u}{C_{0}}\right) \cdot(1+\psi) .
$$

The last observation is correct while $\varphi$ and $\psi$ velocity ratios remain the same as for the stage with typical $\alpha_{1}$ and $\beta_{1}$ angles. Actually, from one hand, $\alpha_{1}$ angle decrease causes a drop of velocity coefficient $\varphi$ itself. From the other hand, lower nozzle outlet angles are favorable since partial admission ratio can be increased towards full-admitted stage decreasing related losses. Therefore, there is a possibility to balance additional losses due to smaller $\alpha_{1}$ angle and finally achieve higher circumferential load $H_{\mathrm{u}}$.

This concept was developed in Saint-Petersburg Polytechnic University (previously Leningrad Polytechnic Institute, LPI) by professors I.I. Kirillov, K.G. Rodin and V.A. Rassokhin since early 1970s. As a result a novel class of highly loaded impulse stages with a high pitch-chord ratio was proposed. These turbine stages, as mentioned by Rassokhin (2004) [13], are characterized by the following features as presented in figure 1:

- small angles $\alpha_{1}\left(3-9^{\circ}\right)$ and $\beta_{1}\left(6-14^{\circ}\right)$;

-high rotor flow turning angle $\left(\Omega=160-170^{\circ}\right)$;

-high pitch-chord ratio for nozzle and rotor blades $\left(t_{1} / b_{1}>1.0, t_{2} / b_{2}>1.1\right)$.
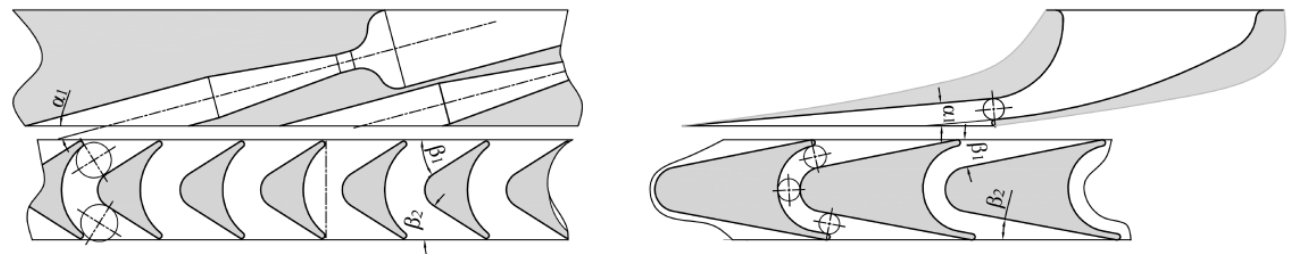

Fig. 1. Comparison of a conventional impulse turbine stage (left) and a stage with high pitch-chord ratio

The advantages of geometrically close rotor blade profiles were outlined by Dorney et al. (2003) [14], Watanabe et al. (2003) [15] and Ghio et al. (2017) [16].

Since exploiting of nozzles with circular cross section is not feasible under the condition $\alpha_{1}<15^{\circ}$ due to technological issues high pitch-chord ratio nozzles have a rectangular cross section. Detailed analysis provided in previous and similar works (see, e.g., Sebelev et al. (2015) [17]) showed that interaction in a supersonic vortex pair formed due to a flow turning in the nozzle vane strongly affects the nozzle efficiency. Thus, the scope of the present work is to provide several new design criteria for such nozzles aimed at diminishing of these vortices interaction and increasing of the high pitch-chord ratio nozzles efficiency. 


\section{Methods}

Present work is based on the results of a numerical simulation for the baseline nozzle and its modifications. Since the proposed modifications do not change the fundamentals of the flow phenomena, the method used is able to provide correct qualitative predictions of the proposed modifications' effectiveness.

Two groups of the modifications are proposed to diminish the losses due to the interaction in the supersonic vortex pair:

- modifications of the nozzle subsonic part and flow inlet direction to discard flow turning and suppress a formation of the vortex pair (series A modifications);

- modifications of the nozzle supersonic part via changing nozzle height-to-throat ratio $l / a_{\mathrm{th}}$ to minimize an interaction in the supersonic vortex pair (series $\mathrm{B}$ modifications).

\subsection{Investigation object and its modifications}

The baseline nozzle is a nozzle of a single-stage axial impulse turbine with rectangular cross section. Design operating conditions are:

- medium - air;

- $p_{0}^{*}=0.37 \mathrm{MPa}, T_{0}^{*}=353 \mathrm{~K}$;

- $p_{2}=0.101 \mathrm{MPa}$.

The main geometrical parameters of the baseline nozzle are provided in table 1 .

Table 1. The main geometrical parameters of the baseline nozzle

\begin{tabular}{|c|c|c|c|c|c|}
\hline Parameter & Dimensions & Value & Parameter & Dimensions & Value \\
\hline$D_{\mathrm{m}}$ & $\mathrm{mm}$ & 300 & $l$ & $\mathrm{~mm}$ & 8.5 \\
\hline$\varepsilon$ & - & 1 & $\mathrm{Z}$ & - & 8 \\
\hline$a_{\mathrm{th}}$ & $\mathrm{mm}$ & 8.77 & $t / b$ & - & 1.02 \\
\hline$\alpha_{1}$ & deg. & 5.0 & $\Delta_{\mathrm{h}}=\Delta_{\mathrm{sh}}$ & $\mathrm{mm}$ & 4.25 \\
\hline
\end{tabular}

\subsubsection{Modifications of a nozzle subsonic part}

The subsonic part of the nozzle was drastically redesigned while implementing series A modifications. The flow inlet direction was switched from axial to tangential (at $\alpha_{1}$ angle). The nozzle walls were contoured in accordance with a Vitoshinski equation, which is usually used for a conjugating of two tubes with different diameters (see Dejch (1970) [18]):

$$
r=\frac{a_{t h}}{\sqrt{1-\left[1-\left(\frac{a_{t h}}{r_{i n}}\right)^{2}\right] \cdot \frac{\left(1-\frac{3 x^{2}}{L^{2}}\right)^{2}}{\left(1+\frac{x^{2}}{L^{2}}\right)^{3}}}} .
$$

Within series A modification three models were proposed - with 1 (A1), 2 (A2) and 3 (A3) contoured walls. These modifications versus the baseline nozzle are depicted in figure 2 . 


\subsubsection{Modifications of a nozzle supersonic part}

Group B modifications aimed at studying of the impact of nozzle height-to-throat ratio $l / a_{\text {th }}$ and nozzle wall curvature ratio $L_{\mathrm{sp}} / R_{\mathrm{m}}$ on the nozzle velocity ratio $\varphi$. First, $l / a_{\mathrm{th}}$ ratio was varied by altering nozzle height $l$ with keeping nozzle throat $a_{\text {th }}$ constant. Mean diameter, number of nozzles and their design also remained constant (B0 series).

Then, in order to establish mutual effect of $L_{\mathrm{sp}} / R_{\mathrm{m}}$ ratio and height-to-throat ratio $l / a_{\mathrm{th}}$ a range of the said parameters was considered with defining the $\varphi$ ratio as a two-variable function. In this, four different nozzle heights $l$ were set for each of three nozzle mean diameters $D_{\mathrm{m}}$ (B1 - B3 series). Thus, $l / a_{\mathrm{th}}$ ratio was altered as described above. $L_{\mathrm{sp}} / R_{\mathrm{m}}$ ratio variation was accomplished by different number of nozzles $\mathrm{Z}$, while nozzle supersonic part length remained constant. The chosen diameters $D_{\mathrm{m}}$ corresponded to the number of nozzle $\mathrm{Z}$ assuming full admission. The principle comparison of the group B modifications versus the baseline is presented in figure 3. All the investigated modifications are presented in table 2 .
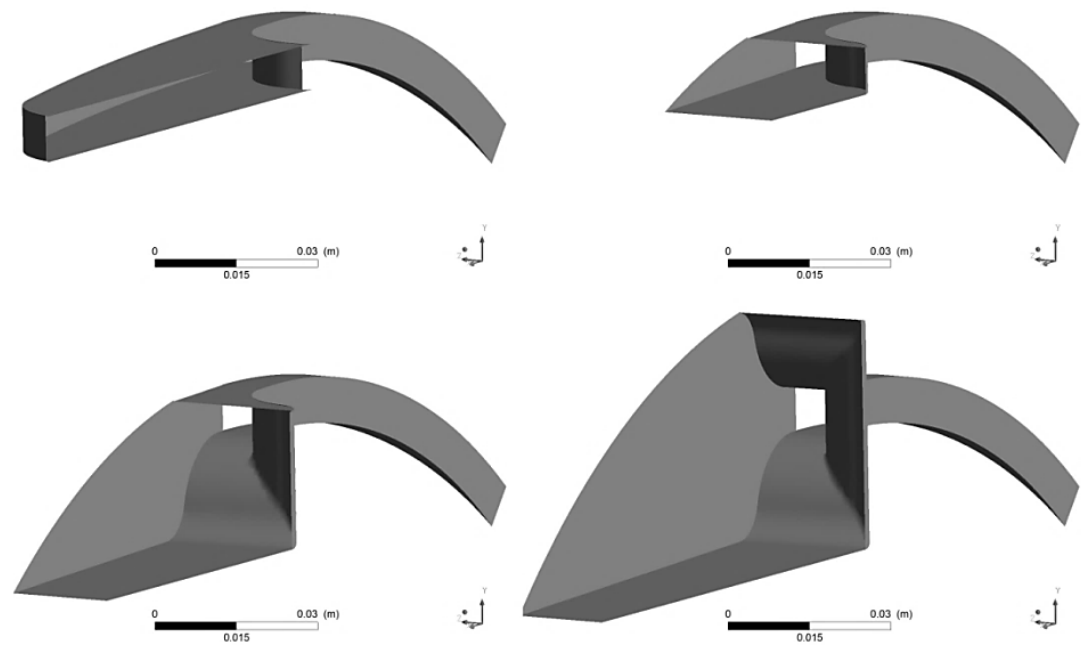

Fig. 2. The baseline nozzle and its series A modifications (from left to right): baseline, A1, A2, A3

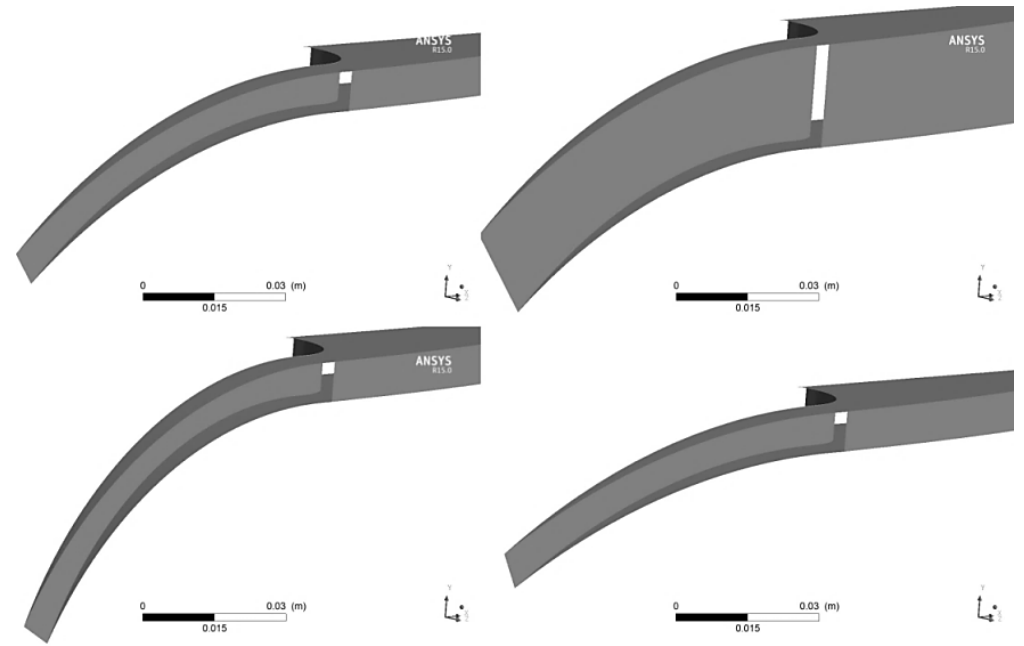

Fig. 3. The principle comparison of the series B modifications versus the baseline nozzle (top left corner) 
Table 2. Variable geometric parameters of the series B modifications

\begin{tabular}{|c|c|c|c|c|}
\hline$D_{\mathrm{m}}, \mathbf{m m}$ & $\mathbf{Z}$ & $L_{\mathrm{sp}} / R_{\mathrm{m}}$ & $l, \mathbf{m m}$ & $l / a_{\mathrm{th}}$ \\
\hline $\begin{array}{c}300.0 \\
\text { (B0 series) }\end{array}$ & 8 & 0.785 & $\begin{array}{l}8.5 ; 12.0 ; 14.6 ; 17.5 ; \\
19.5 ; 22.0 ; 25.0 ; 37.0\end{array}$ & $\begin{array}{l}0.97 ; 1.37 ; 1.66 ; 2.00 \\
2.22 ; 2.51 ; 2.85 ; 4.22\end{array}$ \\
\hline $\begin{array}{c}112.5 \\
\text { (B1 series) }\end{array}$ & 3 & 2.094 & \multirow{3}{*}{$8.5 ; 12.0 ; 19.5 ; 27.0$} & \multirow{3}{*}{$0.97 ; 1.37 ; 2.22 ; 3.08$} \\
\hline $\begin{array}{c}187.5 \\
\text { (B2 series) }\end{array}$ & 5 & 1.257 & & \\
\hline $\begin{array}{c}412.5 \\
\text { (B3 series) }\end{array}$ & 11 & 0.571 & & \\
\hline
\end{tabular}

\subsection{Numerical simulation method}

Steady-state RANS simulations were performed with using ANSYS CFX commercial solver. The computational domain contains 1 nozzle sector, inlet and outlet regions with periodic boundary conditions. The outlet region contains walls which imitate hub and shroud overlaps as shown in figure 4.

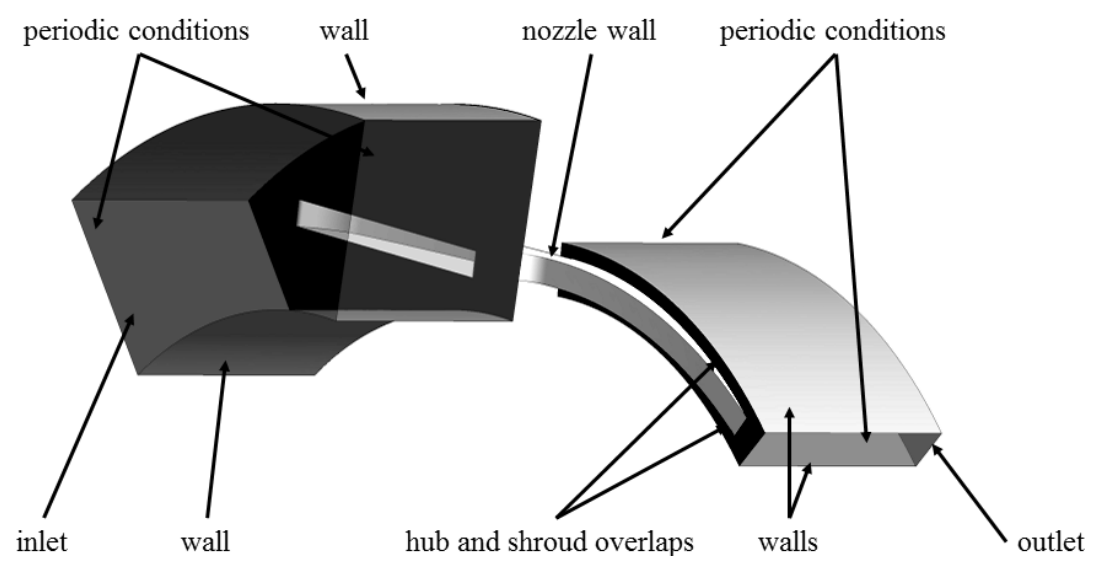

Fig. 4. The computational domain

The pair of total parameters at the inlet - static pressure at the outlet were set as the boundary conditions. k- $\omega$ SST turbulence model with wall functions was used since results performed by Grönman and Turunen-Saaresti (2011) [19] did not show significant difference for the similar problem when wall functions / w/o wall functions approaches were used. The grid size was chosen on the base of the grid independency study performed by Epifanov (2012) [20]. The overall grid size varies from 900k nodes up to 3.5 million nodes depending of the nozzle height and its configuration.

The following equations were used to calculate nozzle overall parameters:

$$
\begin{gathered}
\varphi=\frac{c_{1}}{\sqrt{2 H_{0}}} ; \\
\mu=\frac{G \sqrt{T_{0}^{*}}}{0.0404 l a_{t h} p_{0}^{*}} .
\end{gathered}
$$




\section{Results and Discussion}

\subsection{Series A modifications}

Comparison of the flow parameters obtained for the baseline nozzle and its series A modifications is presented in table 3. Analysis of the results shows that none of the provided modifications exhibit higher efficiency than the baseline nozzle.

Table 3. Simulation results of the series A modifications versus the baseline

\begin{tabular}{|l|c|c|c|c|}
\hline Parameter & $\begin{array}{c}\text { The baseline } \\
\text { nozzle }\end{array}$ & $\begin{array}{c}\mathbf{A 1} \\
\text { modification }\end{array}$ & $\begin{array}{c}\mathbf{A 2} \\
\text { modification }\end{array}$ & $\begin{array}{c}\mathbf{A 3} \\
\text { modification }\end{array}$ \\
\hline$G, \mathrm{~kg} / \mathrm{s}$ & 0.462 & 0.453 & 0.464 & 0.468 \\
\hline$\mu$ & 0.974 & 0.955 & 0.978 & 0.987 \\
\hline$\varphi$ & 0.950 & 0.936 & 0.946 & 0.949 \\
\hline
\end{tabular}

Figure 5 presents velocity flow fields at the nozzles outlet. All the nozzles have hub and shroud spurious counter-rotating vortices formed due to hub and shroud overlaps. These vortices are low-energy. Since the flow at the nozzle outlet has a radial velocity component shroud spurious vortex is limited in space. At the same time a hub vortex occupies all the available space in a hub region.

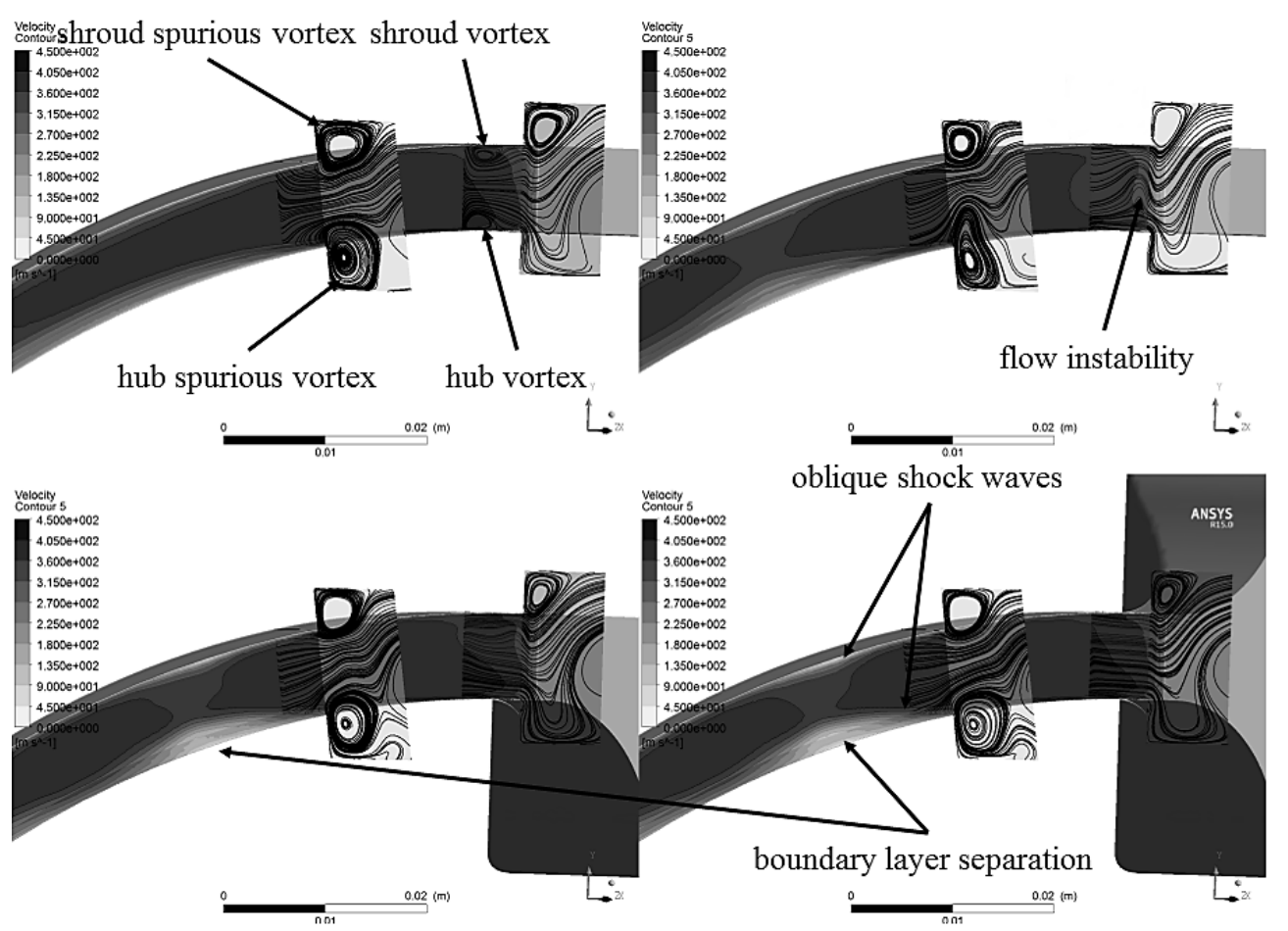

Fig. 5. Velocity fields at the nozzles outlet (from left to right): baseline, A1, A2, A3

The baseline nozzle experiences the mentioned vortex pair structure formation in its flow path while A2 and A3 modifications provide uniform flow structure. A1 modification provides unstable flow distribution. All the nozzles demonstrate typical structure for supersonic nozzle jets (see, e.g., Norman et al. (1982) [21]) characterized by a complex oblique shock wave system and boundary layer separation zones in a hub region caused by 'pulsating' free jet boundary. However, the baseline nozzle has a lower mass flow rate and, 
hence, lower velocities at its outlet compared with its modifications. Physically, it is a consequence of that uniform flow in the nozzle cross section provides higher flow section than in case of a vortex pair presence. Therefore, the baseline nozzle has only one conical shock in its supersonic part while all the modifications have two shocks as shown in figure 5. The last obstacle positively affects the nozzle efficiency, hence, it may be concluded that in the case of interest a vortex pair flow structure in the nozzle flow path is preferable than uniform one.

\subsection{Series B modifications}

Results of the simulations performed for series B modifications are presented in figure 6. For B0, B2 and B3 series the nozzle velocity ratio $\varphi$ maximum is observed near the value $l / a_{\mathrm{th}}=2.39$. This observation positively correlates with the other authors data (Belova et al. (2014) [22]) and indicates growth of the secondary losses when $l / a_{\text {th }}$ ratio is below 2.39 as shown in figure 7 . Physically this means that for $l / a_{\mathrm{th}}>2.39$ the vortex pair transforms to a system of two independent vortices. An incremental decrease of $\varphi$ ratio for $l / a_{\mathrm{th}}>2.39$ is related with a rise of the friction losses due to increase of the friction area at the shroud of the nozzle supersonic part.

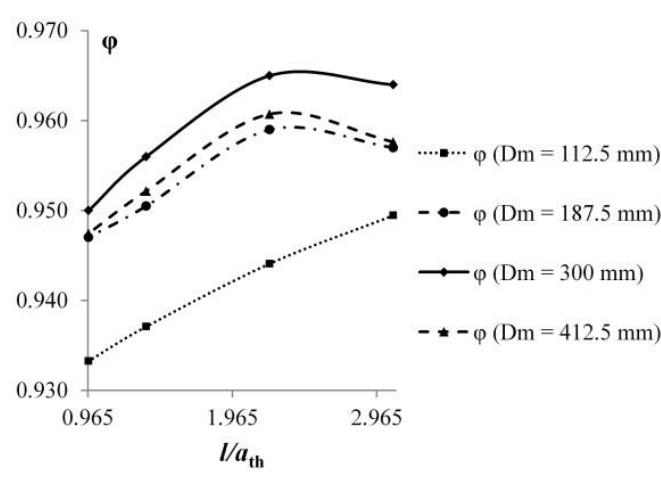

a)

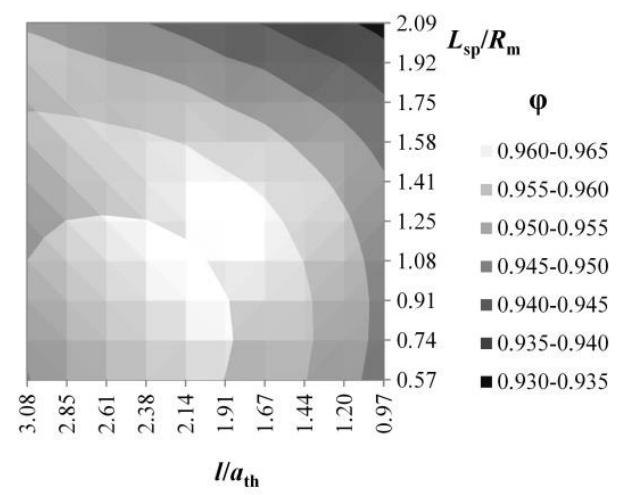

b)

Fig. 6. Results for the series B simulations: (a) nozzle velocity ratio vs $l / a_{\mathrm{th}}$ ratio, (b) approximation of the obtained results for nozzle velocity ratio via regression model

Another effect is that for $\mathrm{B} 1$ series (highest $L_{\mathrm{sp}} / R_{\mathrm{m}}$ ratio) $\varphi$ ratio steadily increases within the whole range of $l / a_{\mathrm{th}}$ ratio. This may be interpreted as follows. According to figure 7 the flow in the nozzle supersonic part of B1 series has a significant separation in the hub region. Increasing of $l / a_{\text {th }}$ ratio leads to decrease of the hub diameter, more intensive flow separation and, hence, drop of the friction losses which positively affects $\varphi$ ratio. Therefore, maximum of $\varphi\left(l / a_{\mathrm{th}}\right)$ function for $\mathrm{B} 1$ series is expected to be for higher $l / a_{\mathrm{th}}$ values.

Effect of $L_{\mathrm{sp}} / R_{\mathrm{m}}$ criteria is related with the flow separation in the nozzle hub region. Figure 7 depicts that occurrence of the latter one crucially affects a turbulence eddy dissipation. According to the regression model $\varphi$ ratio sees dramatic decrease at $L_{\mathrm{sp}} / R_{\mathrm{m}}$ ratio over approximately 1.2 as shown in figure $6 \mathrm{~b}$.

As a consequence, values $L_{\mathrm{sp}} / R_{\mathrm{m}}<1.2$ are preferable as well as values $l / a_{\mathrm{th}}>2.39$ while designing the high pitch-chord ratio nozzles. 

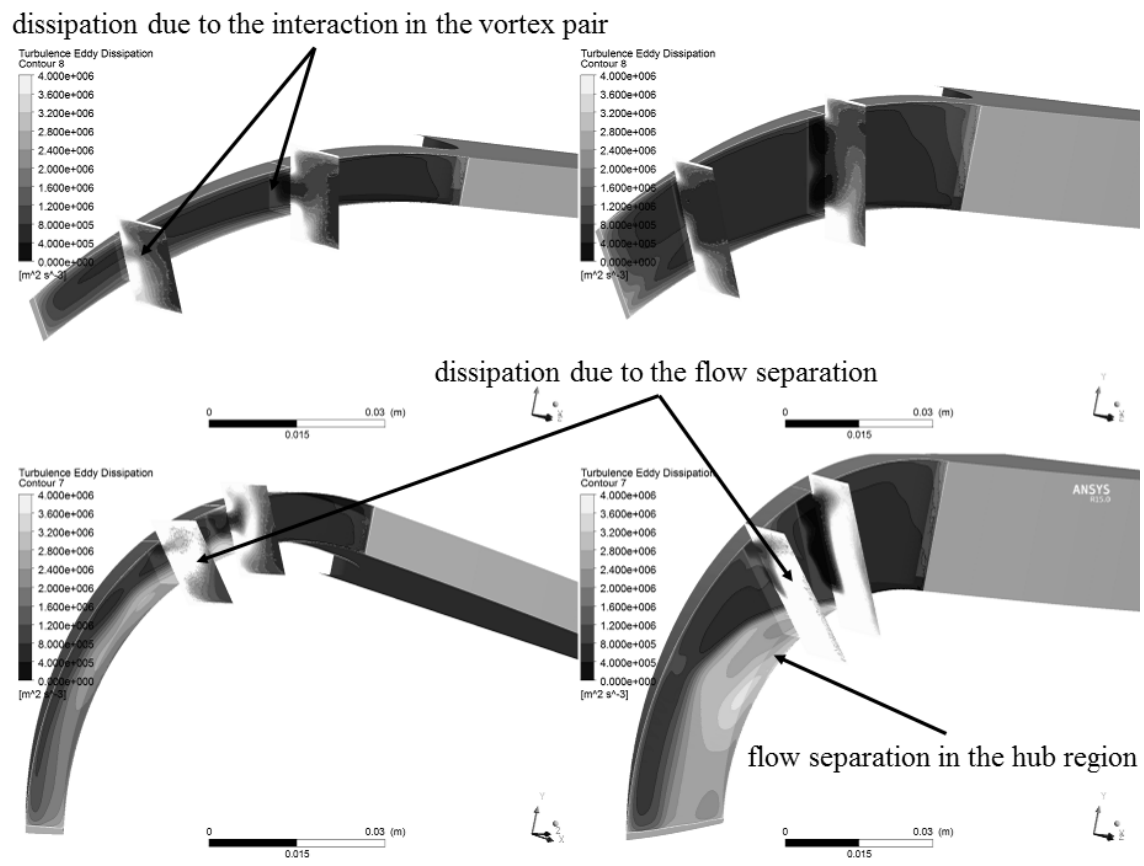

Fig. 7. Turbulence eddy dissipation fields at the nozzles outlet: B0 series (top), B1 series (bottom)

\section{Conclusion}

The scope of the present investigation was in providing of new design criteria for novel supersonic nozzles with a high pitch-chord ratio. Two series of the baseline nozzle modifications were proposed. The first one involved redesign of the nozzle subsonic part to suppress the vortex pair formation. It was shown that in the case of interest a vortex pair flow structure in the nozzle flow path provided higher nozzle velocity ratios than the uniform one. Then, the second series of the baseline modification aimed at establishing optimal configuration of the nozzle supersonic part were introduced. Two design ratios were proposed - nozzle height-to-throat ratio and nozzle wall curvature ratio. The regression model was used to provide optimal values of these ratios.

Authors would like to thank Gennady Rakov for the proposed ideas of the design modifications.

\section{Nomenclature}

$\begin{array}{ll}a_{\mathrm{th}} & \text { nozzle throat }(\mathrm{mm}) \\ c & \text { velocity }(\mathrm{m} / \mathrm{s}) \\ D_{\mathrm{m}} & \text { mean diameter }(\mathrm{m}) \\ G & \text { mass flow rate }(\mathrm{kg} / \mathrm{s}) \\ H_{0} & \text { isentropic enthalpy drop }(\mathrm{kJ} / \mathrm{kg}) \\ H_{\mathrm{u}} & \text { circumferential stage load }(\mathrm{kJ} / \mathrm{kg}) \\ L_{\mathrm{sp}} & \text { length of nozzle supersonic part }(\mathrm{mm}) \\ l & \text { nozzle height }(\mathrm{mm}) \\ p & \text { pressure }(\mathrm{MPa}) \\ T & \text { temperature }(\mathrm{K}) \\ \alpha_{1} & \text { nozzle outlet angle in stationary frame }(\mathrm{deg} .) \\ \beta_{1} & \text { rotor inlet angle in relative frame }(\mathrm{deg} .) \\ \varphi, \psi & \text { nozzle and rotor velocity ratios }\end{array}$

\begin{tabular}{|c|c|}
\hline$r$ & radius coordinate $(\mathrm{mm})$ \\
\hline$t / b$ & pitch-chord ratio \\
\hline$x$ & length coordinate $(\mathrm{mm})$ \\
\hline $\mathrm{u} / \mathrm{C}_{0}$ & stage specific speed \\
\hline $\mathrm{Z}$ & number of nozzles \\
\hline$\Delta$ & overlap value (mm) \\
\hline$\varepsilon$ & partial admission rate \\
\hline$\pi$ & pressure ratio \\
\hline \multirow[t]{3}{*}{$\mu$} & nozzle mass flow ratio \\
\hline & Subscript \\
\hline & $\begin{array}{ll}* & \text { total parameter } \\
\text { h, sh } & \text { hub, shroud }\end{array}$ \\
\hline
\end{tabular}




\section{References}

1. J.W. Kurzrock, Proc. of the ASME Gas Turbine Congress and Exposition, 89-GT-238, (1989).

2. H. Kunte, J.R. Seume, SAE International, 2013-24-0092, (2013).

3. A.S. Natalevich, Vozdushnye mikroturbiny. Mashinostroyenie, Moscow. (1979).

4. D.J. Dorney, L.W. Griffin, K.L. Gundy-Burlet, J. of Prop. and Power, 16, 370-375 (1999).

5. R.A. Tog, A.M. Tousi, Prop. and Power Research, 2(1), 70-83 (2013).

6. A.K. Varma, S. Soundranayagam, Proc. IMechE Part G: J. of Aerosp. Eng., 228(1), 2034 (2012).

7. S. Rashid, M. Tremmel, J. Waggot, R. Moll, Curtis stage nozzle/rotor aerodynamic interaction and the effect on stage performance, J. of Turbomach, 129, (2007).

8. T. Sonoda, T. Arima, M. Olhofer, B. Sendhoff, F. Kost, P.-A. Giess, A Study of advanced high-loaded transonic turbine airfoils, Trans. of the ASME, 128 (2006).

9. T. Wolf, F. Kost, E. Janke, F. Haselbach, L. Willer, Proc. of ASME Turbo Expo 2010: Power for Land, Sea and Air, GT2010-23808. (2010).

10. P. Rajeevalochanam, S.N. Sunkara, B. Mayandi, B.V. Ganesh Banda, V.S.K. Chappati, K. Kumar, Proc. of ASME Turbo Expo 2016: Turbomachinery Technical Conference and Exposition, GT2016-56178 (2016).

11. R.H. Aungier, Turbine aerodynamics: axial-flow and radial inflow turbine design and analysis. ASME press. (2006).

12. I.I. Kirillov, Teorija turbomashin. Mashinostroyenie, Leningrad. (1972).

13. V.A. Rassokhin, St. Petersburg State Polytech. Un. J., 491, 152-161 (2004).

14. D.J. Dorney, L.W. Griffin, F.W. Huber, D.L. Sondak, Proc. of 41th Aerospace sciences meeting \& Exhibit, AIAA 2003-1212, (2003).

15. E. Watanabe, T. Nakano, K. Tanaka, M. Tsutsumi, Y. Tanaka, H. Ohyama, T. Miyawaki, T. Shinohara, Development of new high efficiency steam turbine. Mitsubishi Heavy Industries, Technical Review. 40(4). (2003).

16. R. Ghio, C. Raffaeli, A. Sabattini, V. Dossena, A. Fusetti, A. Spinelli, C. Osnaghi, F. Cozzi, Proc. of 12th European Conf. on Turbomachinery Fluid Dynamics \& Thermodynamics, ETC2017-190, (2017).

17. A. Sebelev, R. Scharf, N. Zabelin, M. Smirnov, Proc. of the 3rd Intern. Seminar on ORC Power Systems, 640-649 (2015).

18. M.E. Dejch, Gazodinamika diffuzorov i vyhlopnyh patrubkov turbomashin. Jenergija, Moscow. (1970).

19. A. Grönman, T. Turunen-Saaresti, Proc. IMechE Part A: J. Power and Energy, 225, 497-503 (2011).

20. A.A. Epifanov, Chislennoe modelirovanie trekhmernogo techeniya $v$ reshetkakh $i$ stupenyakh maloraskhodnykh turbin LPI. PhD thesis. Peter the Great Saint-Petersburg Polytechnic University, Saint-Petersburg, Russia. (2012).

21. M.L. Norman, L. Smarr, K.-H.A. Winkler, M.D. Smith, Astron. Astrophys, 113, 285302 (1982).

22. S.E. Belova, E.N. Bogomolov, S.Yu. Yakovleva, I.A. Nemtyreva, Vestnik PNIPU: Aehrokosmicheskaya tekhnika, 36, 6-21 (2014). 